\title{
LA CIENCIA QUE NO ENSEÑAMOS
}

\author{
CAMPANARIO, JUAN MIGUEL \\ Grupo de Investigación en Aprendizaje de las Ciencias. Departamento de Física. \\ Universidad de Alcalá de Henares. 28871 Alcalá de Henares. Madrid. \\ E-mail: fscampanario@alcala.es
}

\begin{abstract}
SUMMARY
Although it is acknowledged that the history and philosophy of science can help in science teaching, an important part of these contents that are included in the curriculum do not take into account key features of this activity. In this paper I discuss some diverse topics related to realities of current science that usually are not taught to students. Some of such topics are those related to the communication in science, goals and motivation of scientists and ethic problems in science. The goal of the paper is to draw the attention of the people involved in research and in teaching sciences about these topics in order to transmit to students a more accurate vision of current science.
\end{abstract}

\section{INTRODUCCIÓN}

La formación científica correspondiente a los niveles de enseñanza primaria y secundaria debería proporcionar a los futuros ciudadanos adultos los elementos básicos de las disciplinas científicas para que sean capaces de entender la realidad que les rodea y puedan comprender el papel de la ciencia en nuestra sociedad. Asimismo, este primer contacto con la ciencia debería contribuir a que éstos desarrollasen ideas adecuadas sobre la ciencia y el conocimiento científico y a que aplicasen hábitos propios del pensamiento y razonamiento científico en su vida cotidiana. Estos objetivos chocan muchas veces con la realidad. A las quejas tradicionales de profesores e investigadores relativas al bajo nivel de conocimientos de los alumnos y a la persistencia evidente en los alumnos de las famosas preconcepciones tras años de enseñanza formal, hay que añadir un nuevo elemento al que sólo se ha comenzado a prestar atención en los últimos años: se trata de las ideas de los propios alumnos sobre la ciencia, el conocimiento científico y, también, sobre cómo se aprende la ciencia. Estas ideas se recogen en la investigación bajo el epígrafe genérico de concepciones epistemológicas (Ryan y Aikenhead, 1992; Wolff-Michael, 1994; Campanario y Otero, 1998). Tal como demuestran numerosos trabajos de investigación, las concepciones de los alumnos acerca de la validez y fiabilidad del conocimiento científico y cómo éste se articula o acerca de cómo se construye y evoluciona la ciencia son con frecuencia inadecuadas. Este tema ha sido tratado con más detalle por otros autores o por nosotros mismos en otros trabajos y hoy se reconoce casi unánimemente que constituye un obstáculo serio para el propio aprendizaje de las ciencias (Campanario y Otero, 1998; Campanario, Cuerva, Moya y Otero, 1997; Hammer, 1994). 
Incluso personas con formación científica o investigadores cualificados mantienen concepciones inadecuadas sobre la ciencia y el conocimiento científico. Así, por ejemplo, no resulta raro que, con frecuencia, los propios profesores de ciencias tengan una formación deficiente en los aspectos relacionados con la naturaleza de la ciencia y la epistemología, la filosofía o la sociología de la Ciencia (Gustafson y Rowell, 1995; Praia y Cachapuz, 1994; Porlán, 1994; Campanario, 1997b). Enfrentados con los contenidos propios de las disciplinas (científicos), estos aspectos metacientíficos reciben una atención menor en los planes de estudio y en muchas ocasiones deben sacrificarse en beneficio de los contenidos «serios». En la universidad son raras las asignaturas de historia, filosofía o epistemología de la ciencia y, en el caso de existir, suelen concebirse como un mero complemento cultural que se articula generalmente en forma de asignaturas optativas con poco peso en el plan de estudios. Es como si existiera una especie de creencia implícita generalizada de que la historia o la filosofía de la ciencia no contribuyen mucho a la formación del futuro científico. Incluso aspectos tan importantes como la ética científica se olvidan completamente y, según nuestro conocimiento, ni siquiera se abordan de manera generalizada en los cursos de doctorado.

Frente a esta situación se empiezan a tomar las primeras medidas. Ya se reconoce abiertamente la necesidad de abordar otros aspectos en el aprendizaje de las ciencias además de los contenidos tradicionales. Así, por ejemplo, en la etapa de educación secundaria obligatoria en España, la LOGSE establece, entre otros, los siguientes objetivos para el área de ciencias:

a) Elaborar criterios personales y razonados sobre cuestiones científicas y tecnológicas básicas de nuestra época mediante el contraste y evaluación de informaciones obtenidas en distintas fuentes.

b) Reconocer y valorar las aportaciones de la ciencia para la mejora de las condiciones de existencia de los seres humanos, apreciar la importancia de la formación científica, utilizar en las actividades cotidianas los valores y actitudes propios del pensamiento científico, y adoptar una actitud crítica y fundamental ante los grandes problemas que hoy plantean las relaciones entre ciencia y sociedad.

c) Valorar el conocimiento científico como un proceso de construcción ligado a las características y necesidades de la sociedad en cada momento histórico y sometido a evolución y revisión continua.

A pesar de lo positivo de las nuevas tendencias, existe un riesgo grave de transmitir visiones e ideas inadecuadas sobre la propia ciencia, esta vez explícitamente. En parte, ello se debe a que muchas de las formulaciones, en filosofía de la ciencia, se refieren al proceso de creación del conocimiento, a la generación de teorías o leyes o al proceso de descubrimiento, olvidando, por ejemplo, la enorme influencia que tienen los procesos de comunicación en ciencia en la formación y articulación del cono- cimiento científico. Factores personales relacionados con la psicología de los científicos o con los deseos de notoriedad, fama o poder suelen dejarse también de lado o no se tienen en cuenta en las teorías y puntos de vista más extendidos en filosofía de la ciencia. Existe un cierto sesgo a favor de la física en las preferencias de los filósofos de la ciencia y esto tiene consecuencias sobre nuestra forma de concebir otras disciplinas como, por ejemplo, la química, las ciencias biológicas o sanitarias que, en general, ocupan un lugar menor.

Sin duda una parte de la persistencia de los problemas anteriores tiene su origen muchas veces en una cierta falta de atención o de conocimiento por parte de investigadores y educadores de los resultados de las líneas actuales de investigación en dinámica de la ciencia actual. De acuerdo con lo anterior, el objetivo fundamental de este trabajo es llamar la atención de la comunidad docente e investigadora en didáctica de las ciencias sobre algunos de los aspectos poco conocidos de la ciencia actual. No es una casualidad que algunos de estos aspectos poco conocidos, como el fraude o la lucha por la prioridad, resulten, con frecuencia «incómodos» para las visiones simplistas que conciben la ciencia como una actividad pura y desinteresada en la que la verdad es la meta suprema. Estos aspectos se mantienen alejados de los alumnos, aunque hace tiempo que ocupan un lugar destacado en las páginas de debate de revistas tan prestigiosas como Science y Nature. Se desea, además, suscitar un debate sobre la necesidad y conveniencia de una elección cuidada de los contenidos metacientíficos que se incluyen en los programas educativos. Con el fin de conseguir los objetivos anteriores, se revisan los distintos aspectos de la dinámica de la ciencia que pueden ayudar a revelar una cara desconocida $y$, para muchos, oculta de la ciencia que, como indica el título del artículo, rara vez enseñamos a nuestros alumnos.

Entre los aspectos más desconocidos y, a nuestro juicio, merecedores de la atención de los investigadores y profesores cabe citar, en primer lugar, aquéllos relacionados con el papel de la comunicación en ciencia (necesidad de mecanismos de intercambio de conocimientos, papel de las redes informales de científicos o «colegios invisibles», obligación de publicar casi a cualquier costa y la posible utilidad de los productos de dicha publicación). A continuación se abordan diversos temas que inciden en el trabajo y los productos de los científicos (las concepciones metafísicas, el papel del azar en el descubrimiento, la replicación, la nueva forma de hacer ciencia a base de recursos ingentes y equipos enormes de científicos y el componente burocrático y administrativo del trabajo científico). Por último, se tratan aspectos «incómodos» como la lucha por el reconocimiento y la pasión por ser el primero en comunicar un descubrimento o una teoría, las actitudes dogmáticas que, a veces, muestran los propios científicos, el valor y fiabilidad dudosos de gran parte del conocimiento publicado, el fraude y la apropiación de ideas como conductas contrarias a la ética científica y, por último, la pasión por enriquecerse que parece haberse apoderado de una parte de los científicos. 


\section{FUNCIONES Y DISFUNCIONES DE LA CO- MUNICACIÓN EN LA CONSTRUCCION DE LA CIENCIA}

Los mecanismos de comunicación entre científicos son fundamentales para la construcción del conocimiento. Como se explica en los apartados siguientes, la comunicación en ciencia presenta algunos rasgos propios que inciden poderosamente en los resultados del trabajo de los investigadores. A pesar de la enorme trascendencia de los procesos de comunicación en la construcción del conocimiento científico, éste es uno de los aspectos comúnmente olvidados en la enseñanza de las ciencias. Incluso en las carreras universitarias se presta poca atención a los procesos de comunicación científica o, en el caso de que se aborden, se reducen a breves nociones generales sobre la búsqueda de la información. El verdadero aprendizaje acerca de la mecánica de las publicaciones y su importancia en las carreras individuales de los científicos se lleva a cabo, generalmente, de manera implícita o no planeada en el transcurso de la formación del personal investigador.

\section{El sistema de comunicación en ciencia}

Por más trascendental o revolucionario que sea un descubrimiento, no puede considerarse definitivo hasta que abandona el laboratorio donde se ha producido y es dado a conocer al resto de la comunidad académica. Como cualquier científico sabe, la publicación y la lectura de artículos y la búsqueda de información es una tarea a las que se dedica un parte sustancial del tiempo de trabajo. El trabajo científico es un empeño colectivo que, en principio, debe estar abierto al escrutinio por otros miembros de la comunidad investigadora. De la comunicación eficaz en ciencia depende tanto el que los científicos puedan dar a conocer sus trabajos como el que puedan estar al corriente de los nuevos avances en su disciplina.

Las revistas especializadas desempeñan además otro papel en la ciencia: constituyen un foro para que los científicos reclamen su prioridad en el descubrimiento. Precisamente en el origen de las revistas científicas está la necesidad de disponer de un mecanismo que pueda evitar, mediante la difusión y publicación de trabajos, la apropiación de ideas por parte de otros investigadores. Tal fue el origen de la primera revista científica, The Philosophical Transactions of the Royal Society, fundada en 1665 y cuyo primer editor fue el inglés Robert Oldenburg. Con la aparición de esta revista la impresión de los trabajos científicos se convirtió en un medio para su publicación y esto resultó ser más eficaz que la ocultación de los mismos para mantener y reclamar la prioridad sobre las ideas y descubrimientos. El número y variedad de revistas científicas en circulación ha crecido enormemente desde los tiempos de Oldenburg. En 1982, el editor Michael Mahoney estimaba que en el mundo existían unas 40.000 revistas científicas que publicaban un artículo cada 35 segundos (Mahoney, 1982). Fieles a sus orígenes, muchas revistas científicas publican las fechas de recepción y aceptación final de los artículos como un medio para establecer sin ningún género de dudas la prioridad de las contribuciones. Esta precaución no es trivial dado que, como se explica más adelante, en algunas áreas muy competitivas existe una diferencia enorme entre ser el primero y ser el segundo en publicar un descubrimiento, lo que puede traducirse, por ejemplo, en ganar o perder el Premio Nobel.

\section{Los «colegios invisibles»}

La socióloga Diana Crane estudió en los años setenta los patrones de comunicación y trabajo en ciencia y descubrió que, normalmente, el número de científicos que trabajan en una línea determinada suele ser realmente pequeño (Crane, 1972). Según Crane, los científicos que en todo el mundo investigan en un tema concreto suelen conocerse entre sí, aunque tal vez no personalmente, y están al corriente de sus trabajos respectivos. Crane denominó colegio invisible a esta comunidad informal de científicos que trabajan en un mismo tema y que intercambian información.

El colegio invisible actúa, además de como red de comunicación e intercambio, como foro de educación y socialización de los nuevos científicos que comienzan su trabajo en una línea concreta de investigación. Los jóvenes científicos aprenden rápidamente quién es quién en un área determinada y qué investigadores son las autoridades reconocidas por los demás especialistas. Los especialistas que trabajan en un área de investigación determinada a menudo intercambian propuestas previas de trabajo y versiones preliminares de los artículos antes de que se den a conocer por otros canales más formales como pueden ser las revistas especializadas. Los seminarios, congresos y reuniones académicas desempeñan también un papel importante en la formación de los «colegios invisibles» en la medida en que ayudan a la difusión rápida de trabajos de investigación y favorecen su análisis y crítica por otros investigadores. Además, este tipo de eventos contribuye al intercambio de puntos de vista y al establecimiento de contactos y redes de colaboración entre los científicos que trabajan en el mismo tema. A veces los congresos sirven para que se formen alianzas entre científicos a favor o en contra de un enfoque, una teoría o una escuela determinada.

\section{Publica o muere}

En nuestros días la publicación de artículos en las revistas especializadas muchas veces no es un medio de dar a conocer unos resultados y se ha convertido casi en un fin en sí mismo. En los países más desarrollados el número de titulados superiores, incluso de doctores, ha crecido espectacularmente y existe una competencia feroz por ocupar los puestos científicos y docentes en las universidades y centros de investigación. Al mismo tiempo, la especialización creciente del trabajo científico da como resultado que sólo unos cuantos especialistas son capaces de entender plenamente y juzgar el mérito de los trabajos que se publican dentro de una línea de investigación. Ello hace muy difícil que los comités de selección puedan evaluar el contenido de las contribuciones 
científicas, lo que se ha traducido en los últimos años en el uso de indicadores bibliométricos para diferentes objetivos. Estos indicadores bibliométricos han experimentado un desarrollo sin precedentes en el marco de las tecnologías de la evaluación.

Siempre ha sido más fácil evaluar y medir la cantidad de las contribuciones que la calidad de las mismas. Ello ha dado lugar a una tendencia a publicar casi cualquier cosa a toda costa, y que se resume en la frase que da título a este apartado. Son varios los indicadores que demuestran esta tendencia. Por una parte, el número medio de autores que firman los artículos científicos recogidos en las bases de datos ha ido creciendo de manera continua desde hace décadas. Así, por ejemplo, en 1955 el número medio de autores por artículo indexado en las bases de datos del Institute for Scientific Information era de 1,83, mientras que en 1980 era de 2,56 y en 1987 era de 3 . No cabe duda de que una parte de este aumento se explica por la complejidad creciente del trabajo científico que exige más colaboración. Sin embargo, otra de las razones del incremento en el número medio de autores radica en la tendencia a incluir en los artículos a todos los miembros de un equipo, hayan o no participado en el trabajo. Por ejemplo, en muchos grupos de investigación existe la costumbre de que el director del mismo figure en todas y cada una de las contribuciones científicas que se producen.

En 1992 el Institute for Scientific Information publicó una relación de los 20 científicos más prolíficos del mundo. El químico ruso Yury Struchknov encabezaba el ranking con 948 artículos como autor o coautor entre los años 1981 y 1990. Esto significa un artículo cada 3,9 días. El bioquímico inglés Timothy Peters ocupaba el último lugar del «top-20» con un artículo cada 11,3 días (Anderson, 1992). Ciertamente, algunos científicos parecen escribir artículos más rápidamente de lo que la mayoría de nosotros los leemos.

¿Cómo se consigue aumentar el número de publicaciones sin aumentar el trabajo de investigación? Existen diversas técnicas más o menos acreditadas. La más común es la conocida como publicación salami, que consiste en dividir cuidadosamente los resultados de un trabajo de investigación en la mayor cantidad posible de «unidades mínimas publicables». Estas «unidades mínimas publicables» se distribuyen por diversas revistas científicas. Otra técnica consiste en enviar casi los mismos experimentos y resultados, con alguna modificación o estudio adicional prácticamente irrelevante, a varias revistas científicas. Por otra parte, muchos estudios pilotos y pruebas preliminares que, en rigor, no merecerían los honores de ser publicadas, se envían sin ningún remordimiento a revistas menores, que sobreviven publicando este tipo de trabajos.

Una carta de rechazo de una revista nunca es bien recibida por los científicos. Muchos escriben airadas misivas de protesta a los editores de las revistas y, a veces, consiguen que éstos den marcha atrás en su veredicto negativo. En el peor de los casos, un rechazo de un artículo en una revista rara vez da como resultado que el trabajo se archive. Diversos estudios han demostrado que, normalmente, los autores envían sus artículos rechazados, casi intactos, a otras revistas científicas hasta que consiguen finalmente publicarlos. En uno de estos estudios, el editor Stephen Lock descubrió que de una muestra de artículos rechazados por la prestigiosa revista British Medical Journal y publicados en otro sitio, sólo el $20 \%$ fueron modificados tras el rechazo inicial (Lock y Smith, 1986). Otro estudio demostró que el $85 \%$ de los artículos rechazados inicialmente por la revista New England Journal of Medicine fueron asimismo publicados en otras revistas (Cicchetti, 1991). Hay quien piensa que, si un científico está convencido de que sus resultados son realmente valiosos, nada hay de objetable en que intente darlos a conocer a toda costa al resto de la comunidad científica (Campanario, 1998b).

El resultado neto de los procesos anteriores es un incremento notable en la cantidad de literatura científica, lo que se traduce en unas pérdidas cada vez mayores de tiempo por parte de los científicos para localizar la bibliografía relevante en un tema determinado. Muchas veces, para encontrar un resultado interesante, es preciso leer decenas de artículos que aportan poco o nada a un tema. Además, la mera producción masiva ya no sirve para conseguir la promoción académica, de forma que es posible publicar y morir (académicamente, se entiende). Una parte de la solución a este problema consiste en eliminar la necesidad de publicar masivamente para sobrevivir en el mundo académico y en favorecer la calidad frente a la cantidad. Se atisban ya algunos signos positivos. Así, por ejemplo, la Universidad de Harvard sólo admite cinco publicaciones en los currículos de los aspirantes a profesor.

\section{¿Quién lee los artículos científicos?}

El Institute for Scientific Information (ISI) es una institución privada que se encarga, entre otras tareas, de contabilizar las citas que reciben los artículos científicos. Las bases de datos del ISI recogen las citas a los artículos publicados en más de 3.500 de las mejores revistas del mundo. Los análisis de los índices de citas ofrecen algunos resultados sorprendentes sobre el impacto de los artículos científicos. Así, por ejemplo, según el ISI, más del $56 \%$ de los artículos indexados entre 1955 y 1987 no fueron posteriormente citados ni siquiera por sus propios autores (Garfield, 1989, p. 7). Sólo el $10 \%$ de los artículos que fueron citados entre 1961 y 1982 recibieron 10 o más citas, mientras sólo el $3,4 \%$ de dichos artículos fueron citados en 25 o más ocasiones (Garfield, 1985, tabla II). Sólo el 2\% de los más de 32 millones de artículos que fueron citados al menos una vez entre 1945 y 1988 fueron citados más de 50 veces y sólo el $0,05 \%$ de estos artículos fueron citados más de 500 veces (Garfield, 1990). Estos datos incluyen las posibles autocitas por los propios autores en artículos posteriores. En resumen, mientras unos pocos artículos son muy citados, la mayoría de los artículos recibe unas cuantas citas a lo sumo o no son jamás citados. 
La realidad de los datos anteriores ha provocado un intenso debate en los medios académicos. Así, para algunos, los datos anteriores vendrían a demostrar la percepción de Broad y Wade de que una gran parte del trabajo científico que se realiza no sirve de nada y que la presión por publicar a cualquier costa convierte la literatura científica en «un océano de artículos no leídos e ilegibles» (Broad y Wade, 1982, p. 222). Da la impresión de que muchas revistas científicas sirven más a los autores que a los lectores. Por el contrario, otros expertos como el presidente del ISI, Eugene Garfield, consideran que el hecho de que un artículo no sea citado no es necesariamente negativo (Garfield, 1993). Por ejemplo, cualquier científico lee muchos más artículos de los que cita y no puede afirmarse, por tanto, que los artículos que no se citan resulten del todo inútiles. Muchos artículos son utilizados en la enseñanza y no necesariamente han de citarse para que resulten útiles. Seglen incluso ha sugerido que la forma matemática de la distribución del número de citas es característica de una actividad que representa un nivel extremo de actuación y desarrollo (Seglen, 1992). Esta forma de distribución tan desigual se presenta en otras áreas de la actividad humana que exigen una actuación excepcional, como, por ejemplo, el rendimiento en el atletismo de élite. De acuerdo con este punto de vista, la existencia de un número elevado de artículos que son poco citados o que nunca son citados no solo no sería negativa sino que es inevitable.

\section{LOS CIENTÍFICOS EN ACCIÓN}

Existen muchos estereotipos equivocados sobre la realidad del trabajo científico. La mayoría de estos estereotipos están asociados a concepciones simplistas acerca de la ciencia. Como se explica en este apartado, también aquí aparecen aspectos que suelen ser poco conocidos.

\section{Las concepciones metafísicas de los científicos}

Un estereotipo común acerca de la ciencia identifica esta actividad como un dominio libre de cualquier idea mística o metafísica. Los científicos serían, de acuerdo con esta visión, seres racionales que orientan su indagación de acuerdo con la más fría de las lógicas. Sin embargo, como nos recuerda el filósofo de la ciencia Gerald Holton, la ciencia no es tan fría (Holton, 1982). Holton considera que las creencias metafísicas de los científicos constituyen una de las bases de su trabajo. Estas creencias metafísicas pueden tomar, por ejemplo, la forma de «thematas»: ideas generales, trascendentes y dominantes que orientan poderosamente el trabajo de los científicos. Holton considera incluso que las creencias metafísicas constituyen una tercera dimensión de la ciencia, además de la analítica y la empírica (Holton, 1982). Como ejemplos de «thematas» podemos citar ideas generales acerca del conocimiento científico, como la causalidad, la simplicidad y la armonía. La interferencia de este tipo de factores se puede apreciar claramente en la transición de la física medieval a la nueva física y también en nuestros días en el desarrollo de teorías como la mecánica cuántica o el electromagnetismo. No cabe duda de que estas ideas influyen a veces poderosamente y no siempre para bien en la evaluación por parte de los científicos de nuevas teorías y contribuciones científicas (Campanario, 1997a, 1993, 1995).

Otras veces, las concepciones más o menos metafísicas de los científicos toman la forma de numerologías. Desde los tiempos de Pitágoras, las relaciones entre números han ejercido una gran fascinación sobre los científicos. Ciertamente, el hecho de que la realidad se preste a un análisis matemático ya es fascinante en sí mismo. Una de las numerologías científicas más conocidas es la que se refiere al inverso de la constante de estructura fina $(1 / \alpha)$. La constante $\alpha$ relaciona varias constantes universales, como son la carga del electrón, la velocidad de la luz, la constante de Planck y el número $\pi$, de acuerdo con la expresión siguiente:

$$
\alpha=\frac{e^{2}}{c(h / 2 \pi)}
$$

El hecho de que valor de $1 / \alpha$ sea un número adimensional cuyo valor es aproximadamente 137 ha dado origen a no pocas especulaciones en física. Científicos tan famosos y serios como Eddington o Sommerfeld han buscado algún significado profundo a esta coincidencia o han intentado relacionarla con otros tales como el que la suma de los valores numéricos de los caracteres hebreos de la palabra kabala -que sirve para designar el conjunto de misticismos judíos-, también sea 137 (Klotz, 1995). Otro ejemplo: hace años, la prestigiosa revista Nature publicó un artículo en el que se relacionaban las constantes físicas fundamentales con el número $\pi$, e incluso la revista Journal of Physical Chemistry publicó un desgraciado artículo sobre las relaciones numerológicas entre constantes físicas que, para inri de su autor, dependían de las unidades de medida (Klotz, 1995). Las fantasías numerológicas no sólo se dan en campos afines a la metafísica (como la física). También se dan incluso en áreas como la síntesis de las proteínas (Klotz, 1995). Un nuevo ejemplo, esta vez positivo, de la influencia de este tipo de factores en el trabajo científico lo constituyen las relaciones numéricas de Chargaff, que expresaban unas relaciones simples entre las bases que componían la molécula de DNA y que orientaron en cierta medida el trabajo de Watson y Crick cuando intentaban establecer la estructura de esta molécula (Watson, 1970).

\section{La buena suerte también cuenta}

La historia del descubrimiento de la penicilina por Fleming es bien conocida e incluso se explica a los alumnos. Éste es uno de los pocos ejemplos de la historia de la ciencia en que se suele transmitir explícitamente una visión de la misma en la cual factores no previstos desempeñan un papel relevante en un descubrimiento. Sin embargo, la mayor parte de las descripciones del progreso científico suelen hacer hincapié en el «método 
científico» como forma natural de impulsar el avance de la ciencia. Existe una cierta resistencia por parte de los científicos a reconocer el papel del azar en sus descubrimientos. Los principales fílósofos de la ciencia admiten sólo de pasada el papel que desempeña el azar en el avance de la ciencia hasta el punto de que este tema permanece todavía casi sin estudiar. Reconocer que el azar juega un papel relevante en el avance de la ciencia supone, en cierta medida, una contradicción con la idea de ciencia como una actividad completamente bajo control. Precisamente el experimento científico está destinado a «preguntar» a la naturaleza en condiciones controladas y replicables. Además, nos resistimos a otorgar el mismo mérito a un descubrimiento hecho por azar que a un logro conseguido tras un trabajo cuidadosamente planeado.

Sin embargo, la historia de la ciencia está llena de ejemplos que demuestran que a veces el azar es el factor determinante en el descubrimiento científico (van Andel, 1994; Campanario, 1996b). Así, por ejemplo, no son raros los ejemplos en los cuales un objetivo deseado se alcanza casualmente. En estas situaciones, el factor que contribuye a conseguir el objetivo de un proyecto puede ser un error involuntario, una interrupción en el trabajo científico, observaciones inesperadas o la formulación de hipótesis equivocadas. Hay que insistir que, en estos casos, son precisamente estos factores «erróneos» los que posibilitan el descubrimiento deseado (Campanario, 1996b). Otro tipo de situaciones en las que interviene el azar tienen que ver con la realización de descubrimientos accidentales que, en principio, no eran el objetivo deseado del trabajo de investigación. De nuevo, el error o las desviaciones en los métodos de trabajo, las observaciones inesperadas o la interupción involuntaria del trabajo hacen posible estas situaciones.

Desde el punto de vista de la enseñanza de las ciencias la incidencia del azar en el descubrimiento científico plantea algunos problemas interesantes. No parece que la enseñanza tradicional trate de fomentar en los futuros científicos las destrezas mentales básicas para hacer frente a lo inesperado. Así, por ejemplo, muchas veces las prácticas de laboratorio se conciben simplemente como ilustración y es frecuente que los guiones sean cerrados, con todos los desarrollos matemáticos y conceptuales finalizados y con una orientación detallada, tipo receta, de los pasos a seguir, con poco espacio para lo inesperado.

La gran paradoja que subyace al descubrimiento accidental es que es preciso conocer profundamente qué constituye lo normal para poder detectar lo inesperado. Así, mientras una concepción simplista cercana al inductivismo explicaría el descubrimiento accidental haciendo referencia a la gran capacidad de observación del científico que lo realiza, una concepción más adecuada destacaría el papel de los conocimientos previos (y de las expectativas basadas en ellos) en la detección de aspectos en un experimento o una situación que están en conflicto con dichos conocimientos o expectativas.

\section{El mito de la replicación}

Una de las creencias populares sobre la ciencia y el método científico que está más extendida, incluso entre los propios investigadores, es que cualquier nuevo resultado que se publica no se admite por la comunidad académica hasta que es debidamente replicado. Dicha replicación se considera uno de los elementos clave de la fiabilidad del conocimiento científico e incluso algunos autores destacan esta característica en programas de enseñanza de las ciencias, como es el caso del aprendizaje por investigación, (Gil y Valdés, 1996; Gil, Carrascosa, Furió y Martínez-Torregrosa, 1991).

La realidad es muy distinta: son pocos los investigadores que pierden su tiempo repitiendo, sin más, experimentos realizados por otros. Eso es así, en parte, debido a que los editores de las revistas científicas prefieren publicar trabajos originales antes que simples replicaciones de trabajos anteriores. Por otra parte, los organismos gubernamentales no están muy dispuestos a «desperdiciar»su dinero financiando investigaciones cuyo objetivo es la mera repetición de resultados anteriores. La originalidad es uno de los valores más apreciados en la ciencia y pocos científicos están dispuesto a invertir tiempo y recursos repitiendo un trabajo anterior[...] a no ser que los resultados que se intenten repetir resulten demasiado espectaculares o sean demasiado buenos para ser ciertos. Sin embargo la replicación habitual de resultados es la excepción, más que la norma en ciencia (Campanario, 1998b, 1998c). Además, como se indica en el apartado siguiente, el desarrollo de la «gran ciencia», con sus costosos experimentos, ha convertido la replicación en determinadas áreas en una mera invocación académica.

\section{La «gran ciencia»}

Como es sabido, las dimensiones de los modernos aceleradores de partículas son del orden de kilómetros; para su manejo son necesarios decenas de ingenieros, científicos y técnicos y los experimentos que en ellos se realizan suelen durar meses. Como consecuencia, no es raro encontrar artículos en revistas de física de partículas firmados por varios cientos de autores. Mientras que Watson y Crick utilizaron unas fotografías de rayos X, unos cuantos modelos mecánicos y mucha imaginación para proponer su modelo para la molécula de DNA, la herencia más o menos directa de este descubrimiento, el Proyecto Genoma Humano, tiene previsto emplear miles de científicos y ordenadores y millones de dólares en una de las tareas de descubrimiento más ingentes que el ser humano haya emprendido jamás. Estos ejemplos ilustran una nueva forma de hacer ciencia, a una escala antes desconocida, con una inversión tal de medios y de recursos humanos impensable en el pasado. La investigación científica orientada al terreno militar y la investigación espacial son otros ejemplos de lo que se ha dado en llamar gran ciencia.

Uno de los antecedentes de la «gran ciencia», lo constituyen las formas de organización del laboratorio de Lawrence en los años treinta y cuarenta (Sánchez-Ron, 
1992, cáp. 2). En la actualidad, esta forma de hacer ciencia no sólo constituye un cambio cuantitativo en lo que se refiere a los recursos utilizados, también ha dado lugar a diferencias cualitativas en la forma en que se concibe y produce el conocimiento científico. En efecto, cuando los experimentos alcanzan un precio exorbitante que dificulta su replicación, cuando la propia teoría se desarrolla a merced del calendario de los experimentos que se pueden realizar con un coste razonable, hemos de reconocer que algo es «ligeramente» distinto a la concepción tradicional de las relaciones entre teoría y experiencia. Por otra parte, en algunas de las áreas propias de la «gran ciencia», como la investigación en física de partículas, los fenómenos que se estudian están a veces mucho más allá del límite de detección de los aparatos y sistemas disponibles, lo que hace que la conexión entre los resultados de los experimentos y los conceptos explicativos sea muy débil (Moravcsik, 1988).

Esta nueva forma de hacer ciencia debería hacernos reflexionar también sobre el papel de los poderes públicos como motor del trabajo científico. La «gran ciencia» sólo puede llevarse a cabo con unos gastos exorbitantes que sólo puede pagar, hoy por hoy, el estado. No se trata ya de que el estado fomente la investigación científica en este terreno, es que sin el estado no habría posibilidad de desarrollar este tipo de investigación.

\section{El componente burocrático del trabajo científico}

En nuestros días la ciencia se ha institucionalizado y es una profesión más con sus propias normas de acceso y sus reglas implícitas y explícitas. Como una muestra del desarrollo sin precedentes de la ciencia como profesión, cabe citar la estimación de Price realizada en 1968 según la cual el $87 \%$ de los científicos que han existido jamás estaban vivos en ese momento (Price, 1968, p. 7). Una parte cada vez más significativa de los científicos trabajan en el sector privado. El trabajo de estos científicos resulta clave en nuestro sistema económico y muchas veces se mantiene, por su propia naturaleza, fuera del control social o estatal. Además, la ciencia actual ha dejado de ser una tarea solitaria o de equipos reducidos formados por dos o tres personas y unos cuantos ayudantes. Hoy día la parte más importante del trabajo científico se desarrolla en grandes equipos en los que intervienen decenas o, en el caso de la «gran ciencia», incluso centenares de científicos. Aun sin llegar a tales extremos, los equipos se suelen articular en una jerarquía en la que se desempeñan diversos papeles. Los científicos más prestigiosos, o con mayor experiencia, desarrollan con frecuencia papeles meramente administrativos. El «gran jefe» dirige y supervisa un equipo humano numeroso en el que existen diversas líneas de trabajo de la que se encargan los «subjefes». Una parte importante del tiempo del «gran jefe»y de los «subjefes» se dedica a coordinar la política de publicaciones y la de recursos, dado que las publicaciones constituyen un elemento fundamental en la comunicación y en el reconocimiento científicos del equipo. Por otra parte, la búsqueda de recursos se traduce en la presentación casi continua de proyectos de investigación, con las correspondientes memorias de resultados y resúmenes de seguimiento, una tarea que en Estados Unidos ha originado abundantes quejas por parte de los científicos. Así, por ejemplo, Roy estimaba que los directores de equipos científicos dedican casi una cuarta parte de su tiempo a este tipo de tareas (Roy, 1985), una labor burocrática que requiere un personal administrativo que se suele pagar con fondos de los proyectos, con lo cual el ciclo se repite. En el proceso de investigación, nuevos becarios e investigadores jóvenes se incorporan a los equipos o se marchan dependiendo de la coyuntura. Visitantes extranjeros acuden por meses o años a los laboratorios del grupo, mientras que hay que organizar la estancia de investigadores del grupo en otros laboratorios. Todo ello debe compatibilizarse con la asistencia y organización de congresos, seminarios y cursos. No hay que olvidar que aquellos científicos que trabajan en universidades deben hacerse cargo también de su carga docente habitual, la de tiza y pizarra frente a varias decenas o centenares de alumnos universitarios. Además, el «gran jefe» y los «subjefes» deben viajar varias veces al año a otros laboratorios nacionales o extranjeros a coordinar acciones integradas con otros equipos que trabajan en el mismo tema. Todos los miembros del equipo deben leer la cada vez más copiosa producción científica en su campo para mantenerse informados de los últimos desarrollos. Por último, es posible que los miembros más prestigiosos del equipo figuren entre los miembros de los comités editoriales de las revistas del área o sean llamados ocasionalmente para evaluar algún artículo o proyectos como jueces y especialistas, lo cual es un honor, pero también una carga adicional. ¿Es este cúmulo de tareas lo que se nos viene a la cabeza cuando hablamos de «hacer ciencia»?

Desde luego, la descripción anterior cuadra poco con las ideas comunes sobre la ciencia y el trabajo de los científicos y se parece más a la de un trabajo propio de un ejecutivo de cualquier empresa. ¿Quién realiza, pues, los descubrimientos: el «gran jefe» que consiguió el dinero para comprar los aparatos y tiene una visión de conjunto de los proyectos, el becario que purificó las muestras, el científico experto que hizo las mediciones o el investigador extranjero que cedió las muestras y pidió un análisis en profundidad de algo que llamó su atención? La respuesta más probable es: todos a la vez y ninguno en concreto. Nos enfrentamos, sin duda, a nuevas complicaciones derivadas de la naturaleza casi industrial de la ciencia moderna.

\section{LA CARA OCULTA DE LA CIENCIA}

La ciencia suele presentarse al público como una actividad inmaculada en la que hombres esforzados luchan incansablemente por alcanzar la verdad. Las motivaciones de estos hombres para dedicar sus energías a desvelar los misterios de la naturaleza suelen ser, en las visiones tradicionales, totalmente desinteresadas y su único afán sería ayudar a la humanidad. Como se explica en este apartado, también en este aspecto la realidad es muy distinta. 


\section{La lucha por el reconocimiento}

El reconocimiento de sus logros por sus semejantes sigue siendo otra de las fuerzas que impulsan el trabajo de los científicos. Puede afirmarse, pues, que una parte del contrato implícito del científico con la sociedad consiste precisamente en el intercambio de productos mentales y resultados útiles a la sociedad por reconocimiento público por parte de sus colegas. ¿Cómo se expresa este reconocimiento? De muy variadas maneras. Quizá la forma más elemental de reconocimiento es la publicación en revistas académicas de alto prestigio. Como todo científico sabe, un artículo publicado en revistas como Science o Nature asegura a sus autores un impacto y una visibilidad notables. Estas revistas, las más selectivas y prestigiosas del mundo, abarcan todas las áreas del saber y en ellas se publican muchas veces los avances más significativos en las diversas áreas o los descubrimientos más llamativos. Otra forma elemental de reconocimiento al trabajo de los científicos consiste en ser citado por otros autores. Como es sabido, un artículo científico incluye al final una sección de referencias donde se recogen los trabajos y antecedentes previos que han sido comentados o citados en él. Ser citado por otros autores es, en parte, un reconocimiento de la utilidad o el impacto del trabajo propio.

Una forma más sofisticada de reconocimiento es la asignación del nombre de un descubridor a un descubrimiento (Merton, 1985). Muchas leyes, teoremas, ecuaciones y postulados llevan el nombre de su descubridor. Así, hablamos de las leyes de Newton, de la ecuación de Schroedinger y del teorema de Fermat. No cabe duda de que unir el propio nombre a un descubrimiento notable es el sueño de muchos científicos que se aseguran así una cierta forma de inmortalidad. Quizá el medio más explícito de reconocimiento científico sea el nombramiento como miembro de academias y comités científicos o la asignación de premios, medallas y galardones. El Premio Nobel es sin duda la más alta distinción en las disciplinas científicas que existen. La medalla Fields desempeña esta misma misión en matemáticas. Todo este cúmulo de recompensas y premios desempeña un papel notable en la motivación de los científicos para implicarse en su trabajo.

\section{Los primeros tienen ventaja}

Un fenómeno relacionado con el ansia por publicar es el deseo de publicar rápidamente. Desgraciadamente, no siempre está claro quien es el primero en realizar un descubrimiento, por lo que son inevitables las luchas por la prioridad. Los archivos más oscuros de la historia de la ciencia demuestran que algunos de los científicos más eminentes del pasado han dedicado una cantidad considerable de tiempo y esfuerzo a luchas y discusiones con sus competidores por la prioridad de determinados descubrimientos. Estas disputas se han mantenido cuidadosamente ocultas en la historiografía común de la ciencia, de manera que la memoria de los «grandes hombres» se ha preservado limpia de cualquier duda relativa a estos aspectos desagradables. En la lista que recoge los nom- bres de aquéllos que lucharon por la prioridad de algún descubrimiento aparecen celebridades de la talla de Galileo, Newton, Hooke, Cavendish, Watt, Lavoisier, Faraday, Davy, Laplace, Legendre o Gauss (Merton, 1985).

Además de los casos clásicos, existen algunos casos más recientes de competencias feroces por ser el primero en realizar un descubrimiento. El premio nobel James D. Watson describió en su libro La doble hélice la intensa lucha que mantuvieron él y Francis Crick contra el equipo de Linus Pauling para ser los primeros en descubrir a mediados de los años cincuenta la estructura de la molécula de DNA (Watson, 1970). En otro ejemplo de prisa por ser el primero, Francis Collins y Benjamin Lewin sometieron a toda clase de ruegos y presiones a los editores de Science y Cell respectivamente para publicar el descubrimiento simultáneo del gen responsable de la neurofibromatosis. Finalmente los resultados aparecieron el mismo día, con uno de los artículos revisado, aceptado y dado a la imprenta en sólo 17 días, un auténtico récord para el lento sistema de evaluación y publicación científica (Roberts, 1991). Un ejemplo extremo de rivalidad por ser el primero se produjo entre Guillemin y Schally en una larga marcha de 20 años para ser el primero en descubrir las hormonas del crecimiento. El resultado fue que ambos se vieron obligados a compartir el mismo año el Premio Nobel de Medicina (Wade, 1981).

La prisa por publicar rápidamente da lugar a situaciones realmente propias de películas de espías. Para empezar, la investigación en muchos laboratorios se lleva a cabo en secreto, entre estrictas medidas de control interno para evitar fugas de información. En algunas áreas punteras, como la investigación en materiales superconductores, se trabaja todos los días del año, casi sin pausa para evitar que otro grupo rival se anticipe. En esta área precisamente, un conocido científico fue acusado de introducir una errata en un artículo para despistar durante algún tiempo a sus competidores. La errata consistía en citar el elemento $Y b$ en vez del elemento $Y$ entre los componentes de una aleación superconductora (Kolata, 1987). En otras áreas punteras los artículos a veces se envían por fax a las revistas científicas y éstas piden a sus jueces un veredicto por teléfono, sin apenas tiempo para haber revisado el artículo, de nuevo en claro contraste con el lento proceso de publicación «normal».

Otras situaciones derivadas del ansia de ser los primeros son aún más sangrantes. Así, por ejemplo, el investigador español Manuel Perucho denunció amargamente los supuestos manejos de algunos árbitros de la revista Nature destinados, según él, a retrasar a toda costa la publicación de un trascendental artículo suyo sobre el cáncer de colon. El escándalo fue monumental y el editor de la revista, John Maddox, tuvo que reconocer que el trato dispensado a Perucho por los árbitros de su revista no fue justo ni acorde con las normas básicas de conducta científica (Maddox, 1993). Cuando hay de por medio algo tan importante como el Premio Nobel, hasta los científicos más serios parecen perder la compostura. 


\section{La ciencia como seno de actitudes dogmáticas}

Uno de los estereotipos comunes hace referencia al científico como una persona abierta a las novedades y como alguien acostumbrado a razonar desapasionadamente y a discutir teorías y puntos de vista en función únicamente de sus méritos intrínsecos. Sin embargo, la historia de la ciencia está llena de episodios en los que los científicos demuestran actitudes dogmáticas y se resisten al descubrimiento. Los ejemplos de resistencia al descubrimiento por parte de los propios científicos son tantos y tan variados que han merecido la atención de algunos filósofos e historiadores de la ciencia (Barber, 1961; Nissani, 1995). Hemos tenido ocasión de tratar en otros trabajos, con más detalle, los paralelismos existentes entre la resistencia de los alumnos al cambio conceptual y la resistencia por parte de los científicos a aceptar nuevas ideas (Campanario, 1997a, 1998a). Sin ánimo de repetir una vez más los numerosos ejemplos de dogmatismo y resistencia, cabe citar algunos de los datos más significativos disponibles sobre este tema:

a) Numerosas teorías hoy aceptadas como parte integrante del conocimiento científico tuvieron que luchar, en su día, contra el escepticismo de la comunidad científica (Nissani, 1995)

b) Al menos en 12 ocasiones, los autores de trabajos científicos que serían premiados posteriormente con el Premio Nobel encontraron dificultades para que las revistas científicas aceptasen los artículos en que daban a conocer tales trabajos (Campanario, 1995).

c) Al menos 18 de los artículos científicos más citados en la historia de la ciencia fueron rechazados inicialmente por los editores y árbitros de las revistas científicas (Campanario, 1996a).

d) Algunos de los científicos más célebres de la historia se han resistido a aceptar nuevas teorías y puntos de vista, en muchas ocasiones porque cuestionaban sus puntos de vista en ciencia o sus propias teorías (Campanario, 1998b).

La realidad anterior contrasta con el estereotipo común según el cual los científicos son personas desinteresadas que buscan la verdad ante todo. Los celos profesionales, las rivalidades entre escuelas y la cerrazón de algunos grandes científicos han llegado a tener consecuencias dramáticas en el desarrollo de sus disciplinas, por no hablar de las carreras profesionales de sus oponentes menos poderosos o de sus colaboradores (Campanario, 1998b, 1998c).

Los episodios de dogmatismo en ciencia tendrían que plantear algunos problemas de conciencia a aquéllos que defienden la idea de que la enseñanza de las ciencias debería tener como resultado el desarrollo de hábitos de razonamiento rigurosos y desapasionados en la línea de los objetivos que se han citado en la introducción de este artículo. Si el dogmatismo no es un rasgo ajeno a la ciencia, corremos el riesgo de perpetrar una falsificación histórica y seleccionar para la enseñanza sólo aquellos aspectos más de acuerdo con nuestro punto de vista sobre cómo es la ciencia (o cómo debe ser). No es sorprendente que, ante la cantidad y variedad de episodios poco edificantes, algunos autores planteen dudas sobre la conveniencia de utilizar la historia de la ciencia como un recurso en la enseñanza o discutan abiertamente el carácter poco ejemplificante de estos episodios (Brush, 1974).

\section{La fiabilidad del conocimiento científico}

Igual que cualquier otra actividad humana, la ciencia no está libre de errores. El error forma parte de la ciencia hasta el punto de que una parte importante del trabajo científico tiene como objeto evitarlo. La historia de la ciencia puede considerarse como una continua y gigantesca corrección de errores, toda vez que nuevas teorías sustituyen a otras y serán, a su vez, sustituidas en el futuro.

No es éste el lugar para hacer una revisión de los grandes errores científicos. Algunos de estos errores se describen en la bibliografía especializada (Kohn, 1988; Benguigui, 1993; Morrison, 1993). Más preocupante que los errores espectaculares que, por fortuna, pudieron ser finalmente descubiertos, es el hecho de que gran parte del trabajo científico en determinadas áreas parece ser de escaso rigor metodológico. Entre estas áreas destaca la biomedicina. De acuerdo con algunas revisiones publicadas, alrededor del $50 \%$ de los artículos publicados en este campo contiene errores estadísticos, la mitad de ellos considerados como «serios» (Pocock, Hughes y Lee, 1987; Murray, 1988). Es bien sabido que la estadística constituye una de las herramientas matemáticas fundamentales de la investigación en biomedicina y su uso inadecuado puede invalidar las conclusiones que se obtienen de los trabajos. Otro ejemplo: dos expertos estadísticos, Gardner y Bond seleccionaron aleatoriamente 45 artículos publicados en otra importante revista médica, British Medical Journal y concluyeron que 7 no eran aptos desde el punto de vista estadístico (Gardner y Bond, 1990). Una de las técnicas metodológicas de la investigación biomédica, la prueba clínica controlada, parece ser que no siempre se realiza con el debido rigor (Schulz, Chalmers, Grimes y Altman, 1994; Tyson, Furzan, Reisch y Mize, 1983; Grimes y Schultz, 1992). Esta técnica de investigación sirve para probar la efectividad de los nuevos tratamientos y medicamentos. Los errores en el planteamiento y desarrollo de las pruebas clínicas controladas ponen en entredicho las conclusiones que se obtienen.

En contraste también con el estereotipo común que concibe la ciencia como una actividad que se corrige a sí misma, corregir un error científico publicado no siempre es fácil. Por una parte, los editores de las revistas científicas no quieren reconocer que han dejado pasar errores con todas las bendiciones. Además, los editores alegan que temen demandas por parte de los científicos «corregidos», algo que en Estados Unidos puede llevar a la ruina a una revista. Como un ejemplo de lo difícil que resulta corregir errores en ciencia, cabe citar la odisea de 
los químicos Menger y Haim para corregir un artículo erróneo publicado por otros autores en 1989 en la prestigiosa revista Journal of the American Chemical Society, una de las más prestigiosas del mundo (Menger y Haim, 1992). Hasta 1992 no consiguieron estos investigadores publicar la corrección, tras múltiples negativas por parte de distintas revistas, incluso a pesar de que los nuevos jueces y asesores reconocieron que el artículo original era erróneo. Como se explica en la sección siguiente, incluso en el caso de que exista un fraude flagrante, resulta difícil conseguir que las revistas publiquen notas de aviso. Además, al igual que sucede con los artículos declarados fraudulentos, el hecho de que se publiquen correcciones y retracciones no garantiza que éstas sean tenidas en cuenta por el resto de los científicos, que pueden no enterarse y continuar citando positivamente artículos declarados erróneos (Pfeifer y Snodgrass, 1990).

\section{Fraude y plagio: dos conductas contrarias a la ética científica}

Como corresponde a una institución y una actividad cuyo fin declarado es la búsqueda de la verdad, la ciencia se basa en la confianza mutua. Aunque cualquier resultado debe recibirse, en principio, con un cierto escepticismo y debería estar sujeto siempre a ulterior confirmación, los científicos tienden a confiar los unos en los otros y a suponer que las contribuciones científicas se hacen de buena fe, sin ánimo de engañar y con el fin de hacer avanzar la ciencia. Por esta razón, el engaño es una de las conductas más dañinas para la ciencia. Otro de los valores más apreciados en la ciencia es la originalidad y las conductas contrarias a este ideal, como son el robo de ideas o el plagio, que suelen considerarse también impropias del investigador.

La existencia de problemas éticos en la ciencia no es nueva. Incluso algunas de las figuras sagradas, como Ptolomeo, Newton, Pasteur o Mendel han estado bajo sospecha de haber caído en algún tipo de conducta contraria a la ética científica (Kohn, 1988; DiTrocchio, 1993; Broad, 1982; Anderson, 1993). Sin embargo, en los últimos años el incremento de los casos de fraude en ciencia ha adquirido unas dimensiones tales que este problema empieza a constituir una seria amenaza para la credibilidad de los científicos. De hecho, es rara la semana en que las revistas Nature o Science no publican nuevos casos de fraudes o de apropiación de ideas.

El problema de las conductas contrarias a la ciencia ha «pillado», en cierta manera, a la institución con la guardia baja. Acostumbrados a mantener una imagen de bondad más allá de toda duda, los científicos no han sabido reaccionar a tiempo ante los casos de fraude o robo de ideas. Las universidades y centros de investigación prefieren tratar estos asuntos de fraude o apropiación indebida de ideas con la mayor discreción posible para evitar cualquier escándalo que perjudique su reputación y haga más difícil conseguir dinero para investigar. Además, son pocas las universidades que disponen de regulaciones legales para tratar estos asuntos.
Por último, en muchos casos, lamentablemente, las consecuencias del fraude o del plagio han sido más negativas para los que las denuncian que para aquéllos que lo cometen (Rhoades, 1995). El caso es que, ante el número creciente de escándalos, la lucha contra las conductas contrarias a la ciencia se ha institucionalizado en los Estados Unidos. Desde 1993 funciona allí una agencia gubernamental encargada de investigar y perseguir el fraude científico y la apropiación de ideas: la ORI (Office for Research Integrity - Oficina para la Integridad de la Investigación, http://ori.dhhs.gov). De acuerdo con sus directrices, la misión de esta especie de policía es luchar contra «la fabricación o falsificación de datos, el plagio y otras prácticas que se apartan seriamente de aquéllas comúnmente aceptadas en la comunidad científica».

¿Cuál es la incidencia de las conductas contrarias a la ética científica? Según una conocida afirmación de Daniel Koshland, ex editor de Science, el 99,9999\% de los científicos son íntegros (Koshland, 1987). Para los defensores de este punto de vista, los casos de fraude o plagio que se dan a conocer se deben a que, como sucede en cualquier comunidad humana, una pequeñísima fracción de científicos acaba por corromperse. Sin embargo, la opinión de otros muchos investigadores es que los casos de fraude que salen a la luz son realmente una fracción pequeña de los casos que realmente se producen. Los resultados de algunos sondeos son realmente preocupantes: el 36\% de los investigadores encuestados por Kalichman y Friedman admitió haber sido testigos de algún caso de conducta contraria a la ética científica (Kalichman y Friedman, 1992). Los escándalos recientes en los que personajes como Robert Gallo (codescubridor del virus del SIDA) (Cohen, 1993) o David Baltimore (Premio Nobel) (Hamilton, 1991) se han visto implicados en denuncias por prácticas contrarias a la ética científica (aunque en este último caso, el acusado haya sido finalmente exonerado) hacen disminuir, sin duda, la confianza del público en la ciencia.

Las consecuencias del fraude en la literatura científica son difíciles de evaluar. Si, como se indica en la sección anterior, es difícil corregir los errores publicados en revistas científicas, todavía es más difícil alertar a la comunidad científica de la existencia de trabajos fraudulentos. Por ejemplo, en uno de los casos mejor documentados de fraude, de las 30 revistas que publicaron 12 artículos que fueron declarados fraudulentos y otros 48 que fueron declarados cuestionables por una comisión que analizó los trabajos del investigador Robert Slutsky, sólo 18 accedieron a publicar notas de corrección sobre 15 de los artículos (Friedman, 1990). Peor aún, incluso artículos que han sido declarados fraudulentos continúan siendo citados positivamente años después (Kochan y Budd, 1992).

A pesar de que las medidas represivas puedan dar algunos resultados, se admite comúnmente que la lucha contra las conductas contrarias a la ética científica pasa por la educación y la prevención (Miller y Hersen, 1992). Así, por ejemplo, en Estados Unidos, se comienzan a incluir cursos de ética científica en los programas 
universitarios y de formación del personal investigador. La Academia Nacional de Ciencias de los Estados Unidos ha publicado un libro orientado a los jóvenes científicos en el que aborda los problemas éticos (NAS-USA, 1994). También se estiman convenientes cambios en la estructura de la propia ciencia y en la manera en que son recompensados los científicos por su trabajo. Concretamente, un énfasis menor en la necesidad de publicar a toda costa podría contribuir a eliminar este tipo de conductas (Thelen y DiLorenzo, 1992).

\section{La ciencia como negocio para los científicos}

En las últimas décadas se ha agudizado un proceso casi imparable de implicación de los científicos en las empresas y, en algunos casos, de un ansia por el enriquecimiento personal y por participar en los sustanciosos beneficios económicos que la investigación depara en ciertas áreas. No es un fenómeno nuevo: Lord Kelving se hizo rico fabricando y vendiendo instrumentos eléctricos de medida. En la actualidad muchos científicos han seguido su ejemplo y se han dedicado a crear sus propias empresas de consultoría o de investigación, especialmente en áreas relacionadas con la biotecnología o la investigación farmacéutica. Además, cada vez más científicos mantienen intereses comerciales relacionados con sus trabajos de investigación. Krimsky y sus colaboradores examinaron alrededor de 800 artículos en el área de ciencias de la vida que fueron publicados en el año 1992 y descubrieron que, en el 34\% de tales artículos, al menos uno de los coautores tenía algún tipo de relación comercial con la investigación que se describía en el trabajo (propiedad de patentes, relaciones con empresas, etc.) (Krimsky et al., 1996). Otras áreas «calientes» por su relevancia económica son las de nuevos materiales, superconductividad o comunicaciones.

El dinero de las empresas tiene algunos efectos inusitados en los resultados que se publican. Así, por ejemplo, Davidson estudió la relación entre fuentes de financiación y resultados de 107 grandes estudios clínicos publicados en 6 de las revistas más importantes en biomedicina. Este autor dividió la muestra en cuatro grandes categorías dependiendo de qué entidad financió los trabajos (firma farmacéutica u otra fuente) y del resultado del estudio (a favor de una nueva terapia o a favor de la terapia clásica) (Davidson, 1986). Las conclusiones demuestran que el $89 \%$ de los estudios financiados por compañías farmacéuticas favorecían las nuevas terapias frente al $61 \%$ de los estudios financiados mediante otras fuentes. Una interpretación razonable de lo anterior es que una parte de los estudios con resultados negativos no se publicaron. La consecuencia es que, si atendemos globalmente al conjunto de las publicaciones, podemos conseguir una impresión falsa acerca de la efectividad de los nuevos tratamientos médicos.

Por otra parte, existen influencias directas entre la publicación de determinados artículos científicos y los beneficios y pérdidas de algunas empresas químicas o farmacéuticas. Por ejemplo, el valor de las acciones de American Home Products pasó de 1,50 dólares a 61,12 dólares tras la publicación de un artículo en una revista especializada que comentaba favorablemente un nuevo producto antiobesidad de la compañía (Wadman, 1996). La investigación sobre fármacos contra el SIDA es otra de las áreas en que las empresas pueden obtener más beneficios económicos. Ciertamente, en palabras del prestigioso editor médico Drummond Rennie, los artículos que se publican en áreas como medicina, farmacología o biotecnología están «demasiado cerca de la receta del médico» (citado por Barinaga, 1992, p. 618).

La respuesta de la comunidad científica al problema anterior ha sido eminentemente defensiva. Las principales revistas médicas han comenzado a exigir una declaración de «conflicto de intereses» en la cual los autores que envían artículos para su publicación han de hacer pública cualquier relación con industrias o firmas que tengan que ver con productos o tratamientos discutidos en el artículo (Campanario, 1998c). La prestigiosa revista Science también exige desde hace algunos años a los posibles autores que den a conocer cualquier factor, económico o no, que pudiera sesgar sus conclusiones. Algunas voces incluso claman por extender estas medidas a los propios jueces y árbitros científicos que se encargan de revisar y aprobar los artículos que se envían a las revistas para su publicación. Es ésta una de las pocas ocasiones en las que se reconoce abiertamente que factores ajenos al mérito científico intrínseco de un trabajo deben ser tenidos en cuenta a la hora de evaluarlo.

\section{CONCLUSIONES}

En la relación anterior se han evitado deliberadamente algunos aspectos controvertidos de la ciencia y de la relación ciencia-sociedad, como son la interacción y dependencia mutuas entre el ejército y la ciencia o los aspectos discutibles y socialmente cuestionados del uso de la ciencia en problemas como la eugenesia o la clonación humana. Tampoco se tratan otros aspectos más relacionados con la situación de desigualdad entre sexos que existe en ciencia. El objetivo de este trabajo era llamar la atención sobre algunos de los aspectos menos conocidos de la ciencia. Es nuestra opinión que éstos y otros aspectos característicos de la ciencia actual deberían formar parte explícita de los planes de estudio de las carreras de ciencias y, al menos en parte, deberían ser abordados incluso en los niveles de enseñanza secundaria.

Gil ha identificado las cuatro posibilidades clásicas en la enseñanza de las ciencias que fácilmente se llevan a los extremos y que causan, según este autor, más daño que beneficio en los alumnos (Gil, 1994): enseñar conceptos, enseñar métodos, enseñar el papel de la ciencia en la sociedad y enseñar tecnología. Resulta sumamente difícil encontrar el equilibrio entre estas opciones, máxime teniendo en cuenta que en la visión tradicional se presta mucha más atención a los aspectos puramente conceptuales y metodológicos. En la formulación de metas para la enseñanza de las ciencias en los niveles obligatorios 
pueden tenerse en cuenta en cierta medida los cuatro aspectos anteriores prestando atención especial a los tres primeros. Todo ello debe hacerse, naturalmente, partiendo de los condicionantes propios de cada caso y de la realidad previsible de los alumnos que van a cursar las asignaturas, lo cual obliga también a incluir otras metas relacionadas con la naturaleza de la propia ciencia y con el aprendizaje de las ciencias.

El aprender acerca de la propia ciencia, su historia, su realidad y su construcción ha dejado de considerarse un mero complemento útil, o más o menos interesante siempre que haya tiempo, para convertirse en parte de la alfabetización cultural de los ciudadanos (Campanario, 1997a, 1998a). Con este fin se incluyen cada vez más contenidos metacientíficos en los programas educativos y en los libros de texto. Precisamente uno de los objetivos comunes de la enseñanza de las ciencias es que los alumnos conozcan la importancia de la ciencia en nuestra sociedad y sean conscientes de las relaciones entre ciencia y tecnología. Un tratamiento riguroso de estos aspectos puede requerir algún tiempo adicional que justifica la limitación de los contenidos puramente conceptuales y metodológicos.

Se desea, como consecuencia directa de todo lo anterior, que los alumnos desarrollen ideas apropiadas sobre el conocimiento científico y sobre los procesos de construcción del mismo. Lamentablemente, incluso los intentos bienintencionados por incluir estos procesos en la enseñanza explícita de las ciencias suelen centrarse en los aspectos filosóficos y dejan de lado los aspectos sociológicos y humanos. Curiosamente, en la enseñanza de otras disciplinas, como el marketing o la economía, se tienen en cuenta factores ideosincráticos o personales como por ejemplo, los diferentes estilos de concebir la publicidad; en cambio, la actividad científica suele concebirse como algo frío, limpio, desapasionado o como una actividad en la que la implicación personal tiene poca importancia.

No cabe duda de que el tratamiento de los temas que se han abordado en este artículo requiere un esfuerzo adicional de formación por parte del profesor. A las tradicionales carencias que se atribuyen a los profesores de ciencias, como la insuficiencia de su formación didáctica y psicopedagógica, hay que añadir una falta de formación en los ciclos iniciales de los aspectos relacionados con la sociología y la dinámica de la ciencia. Creemos que el interés de los contenidos que se han revisado en este artículo justifica sobradamente el esfuerzo adicional de formación que se solicita.

\section{AGRADECIMIENTOS}

Este trabajo se realizó en el marco del proyecto 023/96 UA financiado con fondos del Consejo Social de la Universidad de Alcalá de Henares. Quiero agradecer las sugerencias y comentarios de un revisor anónimo (algunos de los cuales se han incorporado al artículo) y las opiniones de Hettie Winkel, de la Universidad de Utrecht (Holanda) y de Isabel Niño de Guzmán, de la Pontificia Universidad Católica del Perú. Una versión reducida de este trabajo apareció en las actas del X Congreso de la Asociación de Ensinantes de Ciencias de Galicia (ENCIGA).

\section{REFERENCIAS BIBLIOGRÁFICAS}

ANDERSON, C. (1992). Writer's cramp. Nature, 355, p. 101

ANDERSON, C. (1993). Pasteur notebooks reveal deception. Science, 259, p. 1117.

BARBER, B. (1961). Resistance by scientists to scientific discovery. Science, 134, pp. 596-602.

BARINAGA, M. (1992). Confusion on the cutting edge. Science, 257, pp. 616-619.

BENGUIGUI, G. (1993). Polywater, sociology of an artifact. Social Science Information, 32, pp. 643-667.

BROAD, W.J. (1982). Fraud and the structure of Science. Science, 212, pp. 137-141.

BROAD, W.J. y WADE, N. (1982). Betrayers of the truth: Fraud and deceit in the halls of science. Nueva York: Simon and Schuster.
BRUSH, S. (1974). Should the history of science be rated X? Science, 183, pp. 1164-1172.

CAMPANARIO, J.M. (1993). Consolation for the scientist: Sometimes it is hard to publish papers that are later highy cited. Social Studies of Science, 23, pp. 342-362.

CAMPANARIO, J.M. (1995). Commentary: On influential books and journal articles initially rejected because negative referees' evaluations. Science Communication, 16, pp. 304325.

CAMPANARIO, J.M. (1996a). Have referees rejected some of the most-cited articles of all times? Journal of the American Society for Information Science, 47, pp. 302-310.

CAMPANARIO, J.M. (1996b). Using Citation Classics to study the incidence of serendipity in scientific discovery. Scientometrics, 37, pp. 3-24. 
CAMPANARIO, J.M. (1997a). ¿Por qué a los científicos y a nuestros alumnos les cuesta tanto, a veces, cambiar sus ideas científicas? Didáctica de las Ciencias Experimentales y Sociales, 11, pp. 31-62.

CAMPANARIO, J.M. (1997b). ¿Quiénes son, qué piensan y qué saben los futuros maestros y profesores de ciencias?: Una revision de estudios recientes. Revista Interuniversitaria de Formación del Profesorado. (En prensa, aceptado para su publicación.)

CAMPANARIO, J.M. (1998a). Ventajas e inconvenientes de la historia de la ciencia como recurso en la enseñanza de las ciencias. Revista de Enseñanza de la Física, 11, pp. 5-14.

CAMPANARIO, J.M. (1998b). Peer review as it stands today. Part 1. Science Communication, 19, pp. 181-211.

CAMPANARIO, J.M. (1998c). Peer review as it stands today. Part 2. Science Communication, 19, pp. 277-306.

CAMPANARIO, J.M. y OTERO, J.C. (1998). Más allá de las ideas previas como dificultades de aprendizaje: las pautas de pensamiento, las concepciones epistemológicas y las estrategias metacognitivas de los alumnos de ciencias. (En revisión).

CAMPANARIO, J.M., CUERVA, J., MOYA, A. y OTERO J.C. (1997). El papel de las estrategias metacognitivas en el aprendizaje de las ciencias Ponencia presentada en el $\mathrm{V}$ Congreso Internacional sobre la Enseñanza de las Ciencias. Murcia. Septiembre de 1997.

CICCHETTI, D. (1991). The reliability of peer review for manuscript and grant submissions: A cross-disciplinary investigation. The Behavioral and Brain Sciences, 14, pp. 119-135.

COHEN, J. (1993). HHS: Gallo guilty of misconduct. Science, 259, pp. 168-170.

CRANE, D. (1972). Invisible colleges: Diffusion of knowledge in scientific communities. Chicago: University of Chicago Press.

DAVIDSON, R.A. (1986). Source of funding and outcome of clinical trials. Journal of General and Internal Medicine, 1, pp. 155-158.

DITROCCHIO, F. (1993). Las mentiras de la ciencia. Madrid: Alianza Universidad.

FRIEDMAN, P.J. (1990). Correcting the literature following fraudulent publication. JAMA, 263, pp. 1416-1419.

GARDNER, M.J. y BOND, J. (1990). An exploratory study of statistical assessment of papers published in the British Medical Journal. JAMA, 263, pp. 1355-1357.

GARFIELD, E. (1985). Uses and misuses of citation frecuency. Current Contents, 43, pp. 3-9.

GARFIELD, E. (1989). Citation behavior-an aid or a hindrance to information retrieval? Current Contents, 18, pp. 3-8.

GARFIELD, E. (1990). The most-cited papers of all time, SCI 1945-1988, Part 1A: The SCI Top 100-Will the Lowry method ever be obliterated? Current Contents, 7, pp. 3-15.

GARFIELD, E. (1993). To what extent is the research literature cited? Lowell Hargens and David Bott examine citation rates in sociology. Current Contents, 5, pp. 3-4.

GIL, D. (1994). Relaciones entre conocimiento escolar y conocimiento científico. Investigación en la Escuela, 23, pp. 17-32.
GIL, D., CARRASCOSA, J., FURIÓ, C. y MARTÍNEZTORREGROSA, J. (1991). La enseñanza de las ciencias en la educación secundaria. Barcelona: ICE, Universitat de Barcelona.

GIL, D. y VALDÉS, P. (1996). La orientación de las prácticas de laboratorio como investigación: Un ejemplo ilustrativo. Enseñanza de las Ciencias, 14, pp. 155-163.

GRIMES, D.A. y SCHULZ, K.F. (1992). Randomized controlled trials of home uterine activity monitoring: A review and critique. Obstetrics and Gynecology, 79, pp. 137-142.

GUSTAFSON, B.J. y ROWELL. P.M. (1995). Elementary preservice teachers constructing conceptions about learning science, teaching science and the nature of science. International Journal of Science Education, 17, pp. 589-605.

HAMILTON, D.P. (1991). Verdict in sight in the «Baltimore Case». Science, 251, pp. 1168-1172.

HAMMER, D. (1994). Epistemological beliefs in introductory Physics. Cognition and Instruction, 12, pp. 151-183.

HOLTON, G. (1982). Ensayos sobre el pensamiento científico en la época de Einstein. Madrid: Alianza Universidad.

KALICHMAN, M.W. y FRIEDMAN, P.J. (1992). A pilot study of biomedical trainees' perceptions concerning research ethics. Academic Medicine, 67, pp. 769-775.

KLOTZ, I.M. (1995). Number mysticism in scientific thinking. The Mathematical Intelligencer, 17, pp. 43-51.

KOCHAN, C.A. y BUDD, J.M. (1992). The persistence of fraud in the literature: The Darsee case. JAMA, 43, pp. 488-493.

KOHN, A. (1988). Falsos profetas. Madrid: Pirámide.

KOLATA, G. (1987). Yb or not Yb? That is the question. Science, 236, pp. 663-664.

KOSHLAND, D.E. (1987). Fraud in Science. Science, 235, p. 141.

KRIMSKY, S., ROTHENBERG, L.S., SCOTT, P. y KYLE, G. (1996). Financial interests of authors in scientific journals: A pilot study of 14 publications. Science and Engineering Ethics, 2, pp. 396-410.

LOCK, S. y SMITH, J. (1986). Peer review at work. Scholarly Publishing, 17, pp. 302-314.

MADDOX, J. (1993). Competition and the death of science. Nature, pp. 263-267.

MAHONEY, M.J. (1982). Publication, politics and scientific progress. The Behavioral and Brain Sciences, 5, pp. 220221.

MENGER, F.M. y HAIM, A. (1992). Struggles to correct published errors. Nature, 359, pp. 666-668.

MERTON, R.K. (1985). La sociología de la ciencia 2. Madrid: Alianza Universidad.

MILLER, D.J. y HERSEN, M. (1992). Research fraud in the behavioral and biomedical sciences. Nueva York: John Wiley and Sons.

MORAVCSIK, M.J. (1988). The limits of science and the scientific method. Research Policy, 17, pp. 293-299.

MORRISON, D.R.O. (1993). The rise and fall of the $17-\mathrm{keV}$ neutrino. Nature, 366, pp. 29-32.

MURRAY, G.D. (1988). The task of a statistical referee. British Journal of Surgey, 75, pp. 664-667. 


\section{INVESTIGACIÓN DIDÁCTICA}

NAS-USA (1994). On Being A Scientist. Responsible Conduct in Research. Washington: National Academy of Sciences.

NISSANI, M. (1995). The plight of the obscure innovator in science: A few reflections on Campanario's note. Social Studies of Science, 25, pp. 165-183.

PFEIFER, M.P. y SNODGRASS, G.L. (1990). The continued use of retracted invalid scientific literature. JAMA, 263, pp. $1420-1423$

POCOCK, S.J., HUGHES, M.D. y LEE, R.J. (1987). Statistical problems in the reporting of clinical trials: a survey of three medical journals. New England Journal of Medicine, 317, pp. 426-432.

PORLÁN, R. (1994). Las concepciones epistemológicas de los profesores: el caso de los estudiantes de magisterio. Investigación en la Escuela, 22, pp. 67-84.

PRAIA, J. y CACHAPUZ, F. (1994). Un análisis de las concepciones acerca de la naturaleza del conocimiento científico de los profesores portugueses de la enseñanza secundaria. Enseñanza de las Ciencias, 12, pp. 350-354.

PRICE, J.D.S. (1968). Little science, big science... and beyond Nueva York: Columbia University Press.

RHOADES, L. (1995). Consequences of whistleblowing for the whistleblower in misconduct in science cases. Washington: Research Triangle Institute.

ROBERTS, L. (1991). The rush to publish. Science, 251, pp. 260-263.

ROY, R. (1985). Funding science: The real defects of peer review and an alternative to it. Science Technology and Human Values, 10, pp. 73-81.
RYAN, A.G. y AIKENHEAD, G.S. (1992). Students' preconceptions about the epistemology of science. Science Education, 76, pp. 559-580.

SÁNCHEZ-RON, J.M. (1992). El poder de la ciencia. Madrid: Alianza Editorial.

SEGLEN, P.O. (1992). The skewness of science. Journal of the American Society for Information Science, 43, pp. 628-638.

SCHULZ, K.F., CHALMERS, I., GRIMES, D.A. y ALTMAN, D.G. (1994). Assessing the quality of randomization from reports of controlled trials published in obstetrics and gynecology journals. JAMA, 272, pp. 125-128.

THELEN, M.H. y DILORENZO, T.M. (1992). Academic Pressures, en Miller, D.J. y Hersen, M. (eds.). Research Fraud in the Behavioral and Biomedical Sciences. Nueva York: John Wiley and Sons.

TYSON, J.E., FURZAN, J.A., REISCH, J.S. y MIZE, S.G. (1983). An evaluation of the quality of therapeutic studies in perinatal medicine. Journal of Pediatrics, 102, pp. 10-13.

VAN ANDEL, P. (1994). Anatomy of the unsought finding. Serendipity: Origin, history, domains, traditions, appeareances, patterns and programmability. British Journal of Philosophy of Science, 45, pp. 631-664.

WADE, N. (1981). The Nobel duel. Nueva York: Anchor Press.

WADMAN, M. (1996). Drug company links test journal's policy. Nature, 383, p. 6.

WATSON, J. (1970). La doble hélice. Madrid: Plaza y Janés.

WOLFF-MICHAEL, R. (1994). Physics students' epistemologies and views about knowing and learning. Journal of Research in Science Teaching, 31, pp. 5-30.

[Artículo recibido en diciembre de 1997 y aceptado en diciembre de 1998.] 